\title{
Drilling super-hot horizons of the Larderello geothermal field: insights from noble gases
}

\author{
Gabriella Magro ${ }^{1}$, Enrica Droghieri ${ }^{1}$, and Fabrizio Gherardi ${ }^{1, *}$ \\ ${ }^{1}$ Consiglio Nazionale delle Ricerche, Istituto di Geoscienze e Georisorse (IGG), 56124 Pisa, Italy
}

\begin{abstract}
In the frame of EU-funded DESCRAMBLE project, the Venelle 2 well was deepened down to $2900 \mathrm{~m}$ b.g.l. to test novel drilling techniques, and to improve the knowledge of physical-chemical conditions of super-hot horizons in the Larderello geothermal field, Central Italy. The major seismic reflectors present in the area were the target of the drilling, to verify their potential as geothermal reservoirs of supercritical fluids. No significant fluid entries were observed during the operations. Noble gases were analysed in the emerging drilling fluids to determine whether deepseated fluids with a different signature than those currently discharged by productive wells entered the borehole. Despite the predominant atmospheric signature of the noble gas suite, ${ }^{3} \mathrm{He} /{ }^{4} \mathrm{He}$ values up to $1.59 \mathrm{R}_{\mathrm{a}}$ were measured, and the contribution of a deep component with $\sim 4 \mathrm{R}_{\mathrm{a}}$ was hypothesised by extrapolation of binary mixing trends.
\end{abstract}

\section{Introduction}

Supercritical geothermal systems are very high temperature systems located at depths near or below the brittle-ductile transition zone in the crust, where reservoir fluids are expected to be in the supercritical state (e.g., [1]). Thermodynamic considerations indicate that supercritical geothermal resources may be very favourable for power production, but major technical challenges are associated with characterising, drilling, and exploiting these high temperature systems [2]. Supercritical conditions have been found at the roots of a number of geothermal fields in the USA (The Geysers and Salton Sea), Japan (Kakkonda), Italy (Larderello), Iceland (Krafla), and Mexico (Los Humeros), but no potentially exploitable resources were encountered until now. Aimed at developing novel drilling technologies for a proof-of-concept test of tapping unconventional supercritical geothermal resources, the 3-years UE-funded DESCRAMBLE project was completed on April 2018 with the deepening (on late 2017) of the Venelle 2 well down to the depth of $2900 \mathrm{~m}$ b.g.l.. An extremely high temperature value of more than $500^{\circ} \mathrm{C}$ was estimated at bottom hole, associated with a leak-off pressure of about 300 bar, but the existence of an exploitable reservoir was not proven [3]. In this project, noble gases concentration and isotope composition were measured to trace the contribution of different sources in the fluids released from rocks during the drilling of the Venelle 2 well.

\footnotetext{
* Corresponding author: f.gherardi@igg.cnr.it
} 


\section{Geological context}

Situated in the inner zone of the orogenic belt of the Northern Apennines, Central Italy, the Larderello-Travale is one of the few steam-dominated geothermal fields in the world. Its exploitation dates back to early years of $20^{\text {th }}$ century, and the current installed capacity is more than $800 \mathrm{MWe}$. Fluid production nowadays exclusively occurs from the so-called deep reservoir, hosted in metamorphic rocks of the Basement and Neogene granitoids. Deep magmatic intrusions into a thinned continental crust act as the heat source for the regional thermal anomaly. A noteworthy feature of the geothermal field is the occurrence of major seismic reflectors, the so-called $\mathrm{K}$ - and H-horizons, that widely extend all over the geothermal field at 3 to $6 \mathrm{~km}$ depth [4,5]. Seismic data indicate that the minimum depths of these reflectors occur in correspondence with the emplacement of large ( 3 to $6 \mathrm{~km}$ wide) intrusive bodies within the borders of the currently exploited geothermal areas. The geological and geothermal significance of these horizons is still widely debated. Some authors believe that the K-horizon is the top of one or more permeable reservoirs containing high-P, supercritical fluids [6]. An alternative hypothesis is that the upper K-horizon coincides with the top of the brittle-ductile transition, and that it is associated with overpressured fluids [7]. Moreover, the blow out of San Pompeo 2 well, occurred in 1979 (2800 $\mathrm{m}$ b.g.l.; [8]), points to the existence of fluids at above-hydrostatic pressure in the deepest parts of the geothermal field.

\section{Deepening of Venelle 2 well}

Located near the San Pompeo 2 well [8], Venelle 2 is an existing non-productive well, originally drilled to a depth of $2200 \mathrm{~m}$ and a temperature of $350^{\circ} \mathrm{C}$ in the southern part of the Larderello geothermal field. In the frame of the DESCRAMBLE project [3], Venelle 2 was deepened down to $22900 \mathrm{~m}$ in an area where the pack of seismic reflections corresponding to the so-called H- and K-horizons was particularly thick and shallow. The site was selected because of the high probability of encountering extremely high $\mathrm{P}, \mathrm{T}$ conditions while reducing at minimum the drilling efforts. Drilling operations taken advantage of innovative technologies and materials specifically designed for extreme T,P conditions, and were completed on late 2017 [3]. Both mud (down to about $2709 \mathrm{~m}$ depth) and geothermal waste water treated with a number of organic and inorganic additives (below this level, down to bottom hole) were used as drilling fluids. The well went to a total loss of circulation at $\sim 2707 \mathrm{~m}$ depth, but no significant fluid entries were observed.

\section{Sampling methods and analytical procedures}

The operational protocol for the deepening of the Venelle 2 well considered both real time gas logging (i.e., in situ detection of continuous signals for selected parameters), and the sampling of discrete fluid aliquots for chemical and isotope analyses in the laboratory. All gas samples were obtained by surface degassing, with a constant volume degasser (CVD), of drilling fluids that had been circulated though the drilling apparatus. Continuous gas logging of $\mathrm{CO}_{2}, \mathrm{C}_{1}-\mathrm{C}_{5}$ saturated hydrocarbons, $\mathrm{H}_{2} \mathrm{~S}, \mathrm{H}_{2}, \mathrm{~N}_{2}$, Ar, and $\mathrm{He}$ was ensured by coupling the CVD to both a Dual Fid chromatograph, and a TCD-MicroGC. Due to the specific design of the sampling apparatus, the emerging drilling fluid was always exposed to the atmosphere before sampling/analysis. Noble gas spot samples for mass spectrometric analyses were collected in concomitance with the occurrence of significant positive anomalies in gas concentrations, as detected by gas chromatography during the continuous gas log. Discrete noble gas samples were analysed in the IGG Rare Gas Lab for He, Ne, Kr, 
Xe abundances, and for their isotopic composition. A stainless steel high-vacuum line was used to separate noble gases from reactive gases. Noble gases were then cryogenically separated each other at selected temperatures. The extraction line is connected to both a magnetic mass spectrometer (MAP 215-50) and a quadrupole mass spectrometer (Spectralab 200, VG-Micromass). Resolution for ${ }^{3} \mathrm{He}$ was close to $600 \mathrm{AMU}$ for $\mathrm{HD}-{ }^{3} \mathrm{He}$ at $5 \%$ of the peak. The air ${ }^{3} \mathrm{He} /{ }^{4} \mathrm{He}$ ratios exhibited a reproducibility of better than $10 \%$ over the analysis period. The mass ratios $4 / 20$ measured on air standards by MMS and QMS showed a reproducibility of more than $10 \%$.

\section{Results and discussion}

Due to their inertness, noble gases emerged as the most reliable geochemical parameters to constrain the origin of crustal fluids drained by the Venelle 2 well, under the harsh conditions of borehole drilling (elevated P,T conditions, circulation of fluids contaminated with unknown amounts of inorganic and organic additives).

He relative concentrations measured in parallel by gas chromatography (continuous gas $\log$ ) and mass-spectrometry (spot samples) allowed for a comparison of the relative sensitivity of the two methods. Notably, He was never detected during the continuous log, reflecting the markedly lower sensitivity of gas chromatographic techniques compared to mass spectrometry. Low, but measurable amounts of He were detected instead by spectrometric techniques. He relative concentration was generally below the typical value of air $(5.24 \mathrm{ppm})$, whereas ${ }^{4} \mathrm{He} /{ }^{20} \mathrm{Ne}$ ratios spanned the range 0.318 to 0.253 , typical of air and air-saturated water (asw) values. Due to the large amounts of measured Ar, noble gas concentrations ( $\mathrm{He}, \mathrm{Kr}$ and $\mathrm{Xe}$ ) were normalized to $\mathrm{Ne}$, and corrected for temperaturedriven solubility effects [9]. For instance, inlet temperature of drilling water was $16^{\circ} \mathrm{C}$ during the first step of drilling $\left(2720-2730 \mathrm{~m}\right.$ depth), up to $50^{\circ} \mathrm{C}$ during the $2790-2810 \mathrm{~m}$ depth interval, and between 24 to $38^{\circ} \mathrm{C}$ during the late stage of the perforation.

Despite the low $\mathrm{He}$ abundance and the atmospheric signature of ${ }^{4} \mathrm{He} /{ }^{20} \mathrm{Ne}$ ratio, the presence of deep-seated He likely derived from a ${ }^{3} \mathrm{He}$-enriched source was unequivocally indicated in all the samples by ${ }^{3} \mathrm{He} /{ }^{4} \mathrm{He}$ values between 1 to $1.59 \mathrm{R}_{\mathrm{a}}$ (with $\mathrm{R}_{\mathrm{a}}={ }^{3} \mathrm{He} /{ }^{4} \mathrm{He}$ reference value for the air; Fig. 1). The severe air contamination intrinsically associated with gas extraction device did not allow for the Ne correction of the measured $R / R_{a}$ values, because this correction is very sensitive to the ${ }^{4} \mathrm{He} /{ }^{20} \mathrm{Ne}$ ratio assumed for the atmospheric component $\left(0.318\right.$ for air, variable between 0.253 to 0.280 for air-saturated water at $10^{\circ} \mathrm{C}$ and $30^{\circ} \mathrm{C}$, respectively). Accordingly, the highest measured ${ }^{3} \mathrm{He} /{ }^{4} \mathrm{He}$ ratio of $1.59 \mathrm{R}_{\mathrm{a}}$ should be considered as a sort of minimum reference value for the "real" $R / R_{a}$ value associated with fluids drained during the perforation of Venelle 2 well. Although ${ }^{3} \mathrm{He} /{ }^{4} \mathrm{He}$ values measured on gases extracted from drilling fluids of the Venelle 2 well cannot be precisely corrected for air contamination, and thus any comparison with ${ }^{3} \mathrm{He} /{ }^{4} \mathrm{He}$ values measured on fluids sampled from near producing wells should be undertaken with great caution, it is worth noting that in one of the wells preliminary sampled in the surroundings of the perforation area, ${ }^{3} \mathrm{He} /{ }^{4} \mathrm{He}$ values significantly higher than in the Venelle 2 well have been measured $\left(2.54 \mathrm{R}_{\mathrm{a}}\right.$ instead of $1.59 \mathrm{R}_{\mathrm{a}}$ ). Further to this, the highest ${ }^{3} \mathrm{He} /{ }^{4} \mathrm{He}$ value found in Larderello (3.2 $\mathrm{R}_{\mathrm{a}}$, [10]), was measured in the San Pompeo 2 well, before its blow-out, not far from Venelle 2. Overall, these features were interpreted as an evidence about the highly impermeable nature of the rock volume (and/or of its external envelope) crossed by the perforation, and suggested that a binary mixing with a deep component with $\sim 4 \mathrm{R}_{\mathrm{a}}$ could be hypothesised based on trends of Fig. 1b.

The ${ }^{4} \mathrm{He} /{ }^{20} \mathrm{Ne}$ values close to air-saturated water end-member indicated that the noble gas atmospheric component derived from the mixing between air and air-saturated water 
end members (Fig. 1). This mixing trend was further confirmed by the behaviour of ${ }^{84} \mathrm{Kr}$, a sensitive noble gas tracer of atmospheric contamination (Fig. 2).
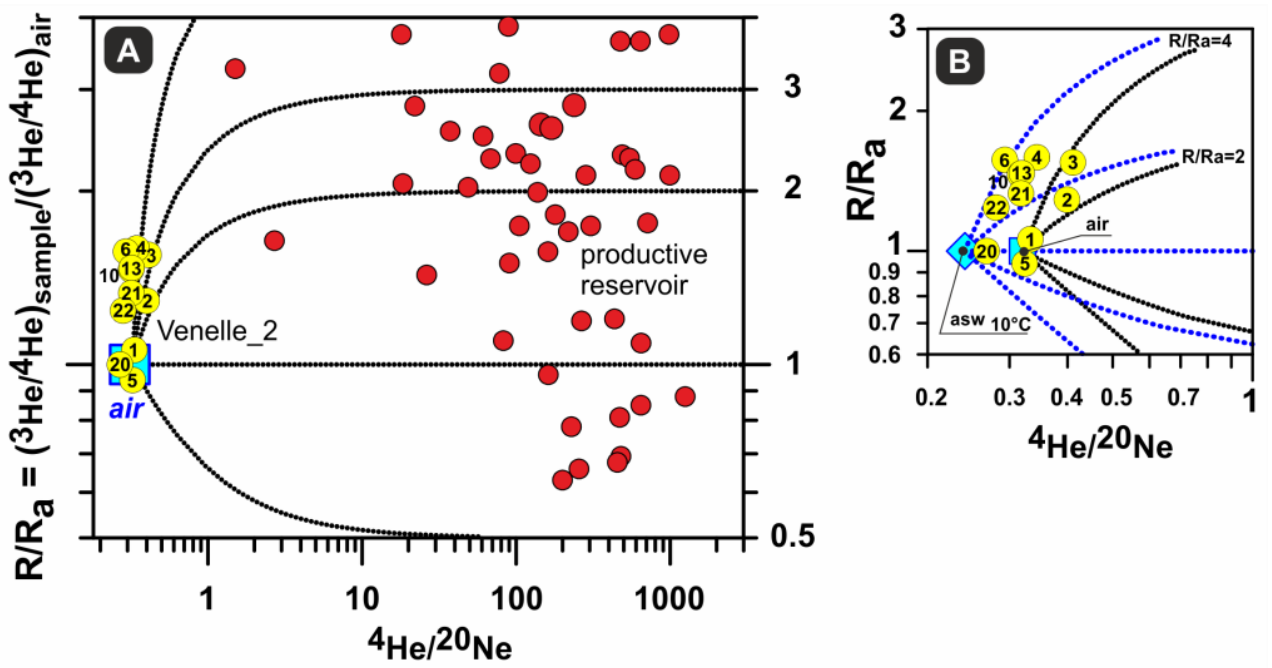

Fig. 1. Box A: R/R $\mathrm{R}_{\mathrm{a}} \mathrm{vs}{ }^{4} \mathrm{He} /{ }^{20} \mathrm{Ne}$ diagram for Larderello gases. Large, yellow dots are samples collected during the deepening of the Venelle 2 well. Small, red dots are samples from productive reservoir. Black curves are binary mixing trends (calculated according to [11]) between the atmospheric component (blue square marked "air") and variably ${ }^{3} \mathrm{He}$-enriched crustal fluids. Box B: zoomed view of the cluster of Venelle 2 samples.

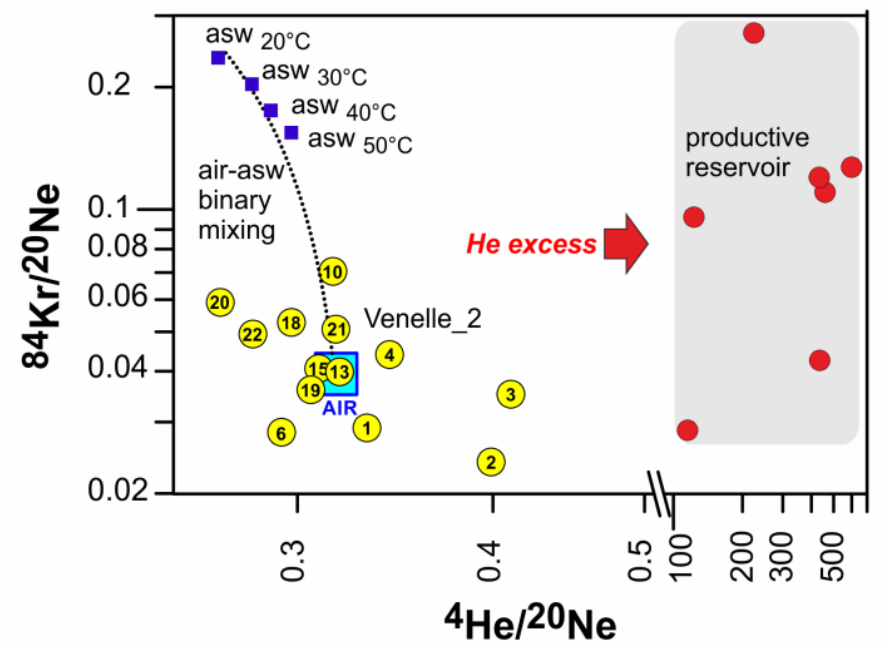

Fig. 2. ${ }^{84} \mathrm{Kr} /{ }^{20} \mathrm{Ne}$ vs ${ }^{4} \mathrm{He} /{ }^{20} \mathrm{Ne}$ diagram for Larderello gases. Symbols are as in Fig.1. The black curve is the binary mixing trend between air and asw.

Generally speaking, the gas phase sampled at the surface consisted of a mixture of air and gaseous compounds mechanically released during the drilling of the well because of the crushing of the rocks. These gases were originally present in the pores of the host rocks, and likely entered the borehole through fissures, either as free gas or as gas dissolved in water. Notably, noble gas signature did not show any trend with depth, coherently with the lithological homogeneity of the host rocks (phyllites), and, most important, independent 
from the distance from seismic reflectors. Even the crossing of the seismic reflectors was not mirrored by any change in noble gases signature.

\section{Conclusions}

Noble gases from Venelle 2 well showed a predominant atmospheric signature, and suggested that during the deepening of the well down to $\sim 2900 \mathrm{~m}$ depth, no major fracture systems were intersected, able to drain fluids from the deepest sectors of the currently productive metamorphic geothermal reservoir, or from other rock volumes possibly acting as reservoirs for supercritical fluids. By comparison of ${ }^{3} \mathrm{He} /{ }^{4} \mathrm{He}$ values measured in the gases extracted from the circulation fluids, with those (relatively ${ }^{3} \mathrm{He}-$ enriched) measured in gases delivered from closely located producing and non-producing (e.g., San Pompeo 2) wells, we speculated that the impermeable boundaries of the rock volume crossed by the drilling were sealed enough to not allow for substantial entry of ${ }^{3} \mathrm{He}-$ enriched fluids already recognized in the surroundings of the borehole. The fact that no relevant changes in the ${ }^{3} \mathrm{He} /{ }^{4} \mathrm{He}$ composition were recorded in concomitance with the crossing of the local seismic reflectors raised fundamental questions about the geothermal, geochemical and hydrological significance of this type of seismic reflectors, starting from their significance as possible targets for drilling and industrial exploitation of supercritical fluids.

We acknowledge the support of the European Union under the Grant Agreement n. 640573 (H2020 Research and Innovation Program, Project DESCRAMBLE).

\section{References}

1. R.O. Fournier. GRL 18, 955-958 (1991)

2. J.W. Tester, B.J. Anderson, A.S. Batchelor, D.D. Blackwell, R. DiPippo, E.M. Drake, J. Garnish, B. Livesay, M.C. Moore, K. Nichols, S. Petty, M.N. Tosköz, R.W. Veatch Jr. MIT 209, 372 pp. (2006)

3. R. Bertani, and the DESCRAMBLE Science and Technology Team. Proc. $43^{\text {rd }}$ Worksh. Geoth. Res. Eng., Stanford, California, February 12-14, 2018, 16 (2018)

4. F. Batini, P.D. Burgassi, G.M. Cameli, R. Nicolich, P. Squarci. Mem Soc Geol It 19, 477-484 (1978)

5. G. Bertini, M. Casini, G. Gianelli, E. Pandeli. Terra Nova 18, 163-169 (2006)

6. F. Batini, G. Bertini, G. Gianelli, E. Pandeli, M. Puxeddu, I. Villa. Geoth Res Counc Trans 9, 1-7 (1985)

7. G.M. Cameli, I. Dini., D. Liotta. Tectonophysics 224, 413-423 (1993)

8. F. Batini, G. Bertini, G. Gianelli, E Pandeli, M. Puxeddu. Mem Soc Geol Ital 25, 219235 (1983)

9. D.R. Hilton. Chem Geol 127, 269-295 (1996)

10. P.J. Hooker, R. Bertrami, S. Lombardi, R.K. O’Nions, E.R. Oxburgh. GCA 49, 25052513 (1985)

11. H. Craig, J. Lupton, Y. Horibe. In Terrestrial Rare Gases (eds. E.G. Alexander jr, M. Ozima), Adv Earth Planet Sci 3, 3-16 (1978) 